\title{
Application of electroreflectance analysis for organic semiconductor thin films
}

\author{
FRANCIS P XAVIER \\ Loyola Institute of Frontier Energy, Loyola College, Chennai 600034, India
}

MS received 20 November 1996; revised 15 April 1997

\begin{abstract}
Many organic semiconductors with conjugate bond structure possess photoconductivity. Conduction mechanism of organic materials exhibits 'dualism' since both intramolecular as well as inter-molecular aspects are involved in the excitation, absorption and transport of charge carriers. Modulation spectroscopy promises to be the most accurate method for analysis of organic photoconductors, especially of thin films. In this technique a periodic perturbation is applied to the material under study and the effect of the perturbation is separated from refiection or absorption while scanning through a given wavelength range by use of lock-in phase sensitive detection method. In electromodulation, particularly in electrolyte electromodulation, the applied field on the material produces changes in the dielectric function which corresponds to the change in reflectance. When the applied field is low the line-shape of spectrum is third-derivative like in comparison with the unmodulated reflectance spectrum. Using Aspnes three-point method the transitions corresponding to critical points can be determined. When the field is intermediate Franz-Keldysh oscillations, which are dc bias dependent, appear on the higher energy side of the transition energy from which the role of intra-molecular as well as intermolecular aspects in conduction mechanism can be understood and the carrier concentration could be determined. Though the electroreflectance method has been developed for inorganic semiconductors, it could be effectively applied for organic/molecular semiconductors as well if the constituent molecules are assumed to be the lattices. The study of organic photoconductors is very important since they are more and more promising especially in photocopying, photovoltaic and solar cells.
\end{abstract}

Keywords. Electroreflectance; Franz-Keldysh oscillations; phthalocyanine.

\section{Introduction}

Some organic compounds having conjugate (i.e. alternate single-double) bonds structure, such as anthracene and phthalocyanine $(\mathrm{Pc})$, have been found to possess semiconducting properties (Inokuchi 1989). Their conductivity and mobility, however, when compared with those of inorganic semiconductors, are very low. A comparison of organic semiconductor (phthalocyanine) properties with those of an elementary semiconductor (germanium) is given in table 1. A few of these organic semiconductors exist in polymorphic modifications. For example, Pcs exist in at least three different forms (Qian 1989; Kanemitsu et al 1991), and differ slightly in structure and electrical properties. The most common polymorphic forms of Pcs are designated $\alpha$-form and $\beta$-form (Moser and Thomas 1963; Gutmann and Lyons 1981; Simon and André 1985). The $\alpha$-form occurs as polycrystalline films when sublimed under vacuum onto cool (room temperature) substrates, while the $\beta$-form is crystallized into single crystals by vacuum sublimation at a temperature around $460^{\circ} \mathrm{C}$ (Xavier and Goldsmith 1995). The $\alpha$-form is tetragonal having six molecules per unit cell but the $\beta$-form is monoclinic having only two molecules per unit cell (Robertson 1935, 1936; Moser and Thomas 1963; Aoyagi et al 1971; Schoch et al 1988). The polymorphs of copper phthalocyanine $(\mathrm{CuPc})$ are compared in table 2 . Perylene interestingly crystallizes in two forms, viz. 
Table 1. Comparison of semiconductor properties: inorganic vs organic semiconductors (Streetman 1980; Heilmeier et al 1963; Gamo et al 1968; Gutman and Lyons 1981; Moser and Thomas 1963).

\begin{tabular}{lll}
\hline & $\begin{array}{l}\text { Inorganic } \\
\text { semiconductor: } \\
\text { germanium }\end{array}$ & $\begin{array}{l}\text { Organic } \\
\text { semiconductor: } \\
\text { phthalocyanine }\end{array}$ \\
\hline Band gap $(\mathrm{eV})$ & $0 \cdot 67$ & 1.6 \\
Mobility $\left(\mathrm{cm}^{2} / \mathrm{V} \cdot \mathrm{sec}\right)$ & 3900 & 1 \\
Carrier concentration $\left(\mathrm{cm}^{-3}\right)$ & $2 \cdot 5 \times 10^{13}$ & $10^{6}$ to $10^{7}$ \\
Trap density $\left(\mathrm{cm}^{-3}\right)$ & & $10^{12}$ to $10^{14}$ \\
Resistivity $(\mathrm{ohm} \cdot \mathrm{cm})$ & 43 & $10^{14}$ \\
Dielectric constant & 16 & $3 \cdot 8$ \\
Density $\left(\mathrm{g} / \mathrm{cm}^{3}\right)$ & $5 \cdot 32$ & $1 \cdot 6$ \\
\hline
\end{tabular}

Table 2. Polymorphs of copper phthalocyanine (Aoyagi et al 1971; Robertson 1935, 1936).

\begin{tabular}{lcc}
\hline & $\begin{array}{c}\text { CuPc } \\
\alpha \text {-form }\end{array}$ & $\begin{array}{l}\text { CuPc } \\
\beta \text {-form }\end{array}$ \\
\hline Cell constants: & & \\
$\mathrm{a}(\AA)$ & 17.37 & 17.34 \\
$\mathrm{~b}(\AA)$ & $3 \cdot 7$ & 4.81 \\
$\mathrm{c}(\AA)$ & 12.79 & 14.66 \\
$\beta$ & & $105.37^{\circ}$ \\
Molecules $/ \mathrm{cell}^{3}$ & 6 & 2 \\
Density $\left(\mathrm{g} / \mathrm{cm}^{3}\right)$ & 1.49 & 1.63 \\
\hline
\end{tabular}

$\alpha$-perylene having pairwise packing and $\beta$-perylene having the herringbone packing. The different molecular packing significantly modifies the physical properties (Wright 1989).

The conductivities of solid complexes made up of polycyclic aromatic hydrocarbons and halogens have been reported in the range $1-10^{-3} \Omega^{-1} \mathrm{~cm}^{-1}$ (Wright 1989). For example, halogen complexes of Pcs are still topic of strong interest in application oriented research (Inabe et al 1985; Xavier and Goldsmith 1995). Electrons associated with conjugate bonds of organic compounds are not localized on a particular atom but are located over the entire molecule. These electrons are in the p-state and hence are called the pi-electrons (Xavier 1993). These highly-conducting complexes have two features in common: (i) they contain planar pi-donor and/or acceptor molecules in segregated stacks and (ii) they contain unpaired electrons which can move along these stacks much more easily than between them. These pi-electrons are responsible for conduction in organic semiconductors (Eley 1989; Inokuchi 1989; Karl 1989). When a pi-electron is excited into a 'free' state, a hole is left behind in the molecule. It is these holes and electrons (electron-hole pair) which participate in the conduction process (Xavier and Pragasam 1996). Further, the overlap between adjacent molecules is highly anisotropic (table 2), leading to the formation of one-dimensional energy bands rather than the usual three-dimensional energy bands, as in cases of conventional inorganic semiconductors (Wright 1989). And even poorly-conducting molecular crystals exhibit 
photoconduction which have resulted in applications for photocopying (Weigl 1977), and photo-electrochemistry (Wrighton 1979), as photovoltaic cells (Chamberlain 1983) and solar cells (Orti et al 1990). Pcs find applications in textile and pain industries as dyes (Orti et al 1988) and they are expected to serve as active materials for molecular electronics such as chemical sensors, etc (Minami et al 1987). Anthracene micro crystals may be used as media for optical signal transfer in molecular structures (Aviksoo and Reinot 1992). As in the case of Pcs the thin films have more molecules per unit cell than that of single crystals and hence organic semiconductor thin films, which are in general good photoconductors, are good candidates especially for solar cells.

Compounds such as polyacetylene and polyacene (Tanaka et al 1983) exhibit metallic conductivity. Recently ambient-pressure organic superconductors have been developed, such as (TMTSF) ${ }_{2} \mathrm{ClO}_{4}$ and the bis (ethylenedithio) tetrathiafulvalene (BEDT-TTF) salts (Hsu et al 1974; Baughman et al 1977; Kivelson et al 1983; Wright 1989).

\subsection{Modulation spectroscopy}

The most accurate method for analysis of organic photoconductor thin films is by using modulation spectroscopy. Modulation spectroscopy involves the application of a periodic perturbation to the material under study while scanning through a given wavelength range and then separating the effect of the perturbation from the reflection or absorption background through lock-in phase sensitive detection in order to measure the periodic modulation of the optical properties of the material (Aspnes et al 1968). The periodic perturbation may be produced by an electric field (electromodulation), light (photomodulation), strain (piezomodulation), heat pulse (thermomodulation), small wavelength disturbance (wavelength modulation), and magnetic field (magnetomodulation) (Xavier 1993). Modulation spectroscopic technique results in sharp, better separated, more richly structured (Aspnes et al 1972) and well-resolved reflection/absorption spectra, with derivative-like structures, that can be analyzed to yield directly the properties of the material under study (Aspnes 1973) such as band gap energy, carrier concentration, impurity effects, Franz-Keldysh oscillations, etc. The unique line shape is caused by the acceleration of the electron induced by the electric field (Poras 1993).

1.1a Electromodulation: In electromodulation the applied electric field on the material of interest produces changes in the dielectric function associated primarily with interband transitions at the critical points (Bordas 1976). Further, electromodulation can destroy the translational symmetry of the material and hence can accelerate unbound electrons and/or holes (Pollak and Shen 1993). Thus the electromodulation is of greatest importance in studying the band structure of a semiconductor. This technique provides optimum sensitivity for the detection of weak or closely spaced critical points. Under the influence of an electric field the energy bands of the semiconductors are tilted and an electron, in order to tunnel from the valence band to the conduction band, meets with a triangular barrier. Further, as the electron tunnels through the barrier, if it interacts with a photon, the effective width of the barrier becomes smaller. As a result of this photon-assisted tunneling the transmission probability becomes exponential. Therefore, oscillatory behaviour is seen in the $\mathrm{d} R / R$ vs photon energy data (Pollak and Shen 1993). These are Franz-Keldysh oscillations. 


\section{Electroreflectance}

Electroreflectance has probably become the most effective of all modulation techniques, due mainly to the simplicity of the electrolytic method (Cardona et al 1967). The theoretical formulation was due to Franz (1958) and Keldysh (1958) which was further extended by Callaway (1963), Tharmalingham (1963), and Aspnes (1966). Electrolyte electromodulation techniques on thin films could be used to gain futher insight into details of the band structure of the material under study. This method is very successful especially when electroreflectance on single crystals is very difficult owing to their small geometric cross section and when photoreflectance is difficult due to lack of high surface reflectivity of thin films under investigation.

Reflectance measurement primarily concerns the surface of the material under study and probes changes in the concentration of carriers. The conventional reflection measurements can be advanced by modulation techniques. Modulation spectroscopy deals with measurements and interpretation of change in optical spectra of a material caused by modifying in some way the measurement conditions (Aspnes 1980). In ER the applied electric field on the sample produces changes in the dielectric function associated primarily with interband transitions (Bordas 1976).

An outline of the mathematical treatment, as given by Aspnes (Aspnes et al 1968; Aspnes 1972, 1973, 1974), for determination of band gap energy from ER spectra is given below (Xavier 1993).

The Fresnel reflectance expression for a two-phase system with light normally incident on the surface is (Aspnes 1980)

$$
R=\left|\left(n-n_{\mathrm{a}}\right) /\left(n+n_{\mathrm{a}}\right)\right|^{2},
$$

with $n^{2}=\varepsilon, n_{\mathrm{a}}{ }^{2}=\varepsilon_{\mathrm{a}}, n_{\mathrm{a}}$, refractive index of ambient, and $\varepsilon_{\mathrm{a}}$, dielectric constant of ambient. Then we can write

$$
\begin{aligned}
\mathrm{d} R / R & =\operatorname{Re}\left[2 n_{\mathrm{a}} \cdot \mathrm{d} \varepsilon / n\left(\varepsilon-\varepsilon_{\mathrm{a}}\right)\right] \\
& =\operatorname{Re}[(\alpha-i \beta \cdot \mathrm{d} \varepsilon)] \\
& =\alpha \cdot \mathrm{d} \varepsilon_{1}+\beta \cdot \mathrm{d} \varepsilon_{2},
\end{aligned}
$$

with $\mathrm{d} \varepsilon=\mathrm{d} \varepsilon_{1}+i \mathrm{~d} \varepsilon ;$ and $\alpha$ and $\beta$, Seraphin coefficients (Seraphin et al 1966). Thus the modulated reflectance is related to the field induced change in the dielectric constant of a material.

Consider now the absorption of light by a solid in the absence of an electric field. Then the perturbation introduced by the photon is given by (Evans 1976)

$$
H_{\mathrm{ml}}^{\prime}=(i e \hbar / m c) \int \Psi_{\mathrm{m}}^{*} \exp (i \mathbf{k} \cdot \mathbf{r}) \mathbf{A} \cdot \nabla \Psi_{1} \mathrm{~d} \tau,
$$

where $\mathbf{A}=\varepsilon A \exp (i \mathbf{k} \cdot \mathbf{r}-i \omega t)$, with $\mathbf{A}$ the vector potential; and $\Psi_{\mathrm{m}}$ and $\Psi_{1}$, the initial and final state wave functions respectively.

Assuming that the one-electron state of the solid are non-degenerate, they can be represented by Bloch function satisfying the equation

$$
\left[\left(-\hbar^{2} / 2 m\right) \nabla^{2}+V(\mathbf{r})\right] \Psi_{n}(\mathbf{k}, \mathbf{r})=E_{n}(\mathbf{k}) \Psi_{n}(\mathbf{k}, \mathbf{r}),
$$

with $V(\mathbf{r})$, periodic in lattice. Using time-dependent perturbation theory the theory of transition rate over the entire solid from the filled discrete state to the empty discrete 
state can be obtained by summing over all filled and empty states. And including the factor 2 for spin degeneracy the transition rate per unit volume per unit time, $W$, is given by (Aspnes et al 1968)

$$
W=\left(2 \pi e^{2} A^{2} / m^{2} c^{2} \hbar\right) \sum_{n^{\prime}, n}\left[2 /(2 \pi)^{3}\right] \int_{\text {B.z. }} \mathrm{d}^{3} k\left|\varepsilon \cdot \mathbf{P}_{n^{\prime}, n}(\mathbf{k})\right|^{2} \delta\left[E_{n^{\prime}, n}(\mathbf{k})-\hbar \omega\right],
$$

with $\mathbf{P}$, momentum matrix element and the integral is over the Brillouin zone. The transition rate is related to the absorption coefficient $(\alpha)$ which in turn is related to the imaginary part of the dielectric constant as given below:

$$
\begin{aligned}
\varepsilon_{2}(\omega) & =n c \alpha / \omega \\
& =\left(2 \pi c^{2} \hbar / \omega^{2} A^{2}\right) W,
\end{aligned}
$$

and the imaginary part of the dielectric constant is then given by

$$
\varepsilon_{2}(\omega)=\left(4 \pi e^{2} / m^{2} \omega^{2}\right) \sum_{n^{\prime}, n} \int_{\text {B.z. }} \mathrm{d}^{3} k\left[2 /(2 \pi)^{3}\right]\left|\varepsilon \cdot \mathbf{P}_{n^{\prime}, n}(\mathbf{k})\right|^{2} \delta\left[E_{n^{\prime}, n}(\mathbf{k})-\hbar \omega\right] .
$$

When an electric field (F), which is a periodic perturbation, is applied to the solid, a change in dielectric function of the solid is induced. The Hamiltonian of the system now is given by

$$
H=H_{0}+H^{\prime}
$$

with $H_{0}=\left(p^{2} / 2 m\right)+V(\mathbf{r})$; and $H^{\prime}=e \mathbf{F} \cdot \mathbf{r}$. Under an electric field the electrons get accelerated. This leads to a change in $\varepsilon$ and consequently in $R$, the reflectance. The applied field is considered low-field range when

$$
|h \Omega| \leqslant \Gamma / 3,
$$

with $\Gamma$, the broadening parameter; and intermediate field range when

$$
|\hbar \Omega| \leqslant \Gamma,
$$

where $h \Omega^{3}=\left(e^{2} \mathbf{F}^{2} \hbar^{2} / 8 \mu\right)$ with $\mu$, interband reduced mass of the conduction, $c$, and valence, $v$, bands evaluated in the field direction and $h \Omega$, characteristic energy obtained in the quantum mechanical solution of a particle of mass $\mu$ (Aspnes 1973).

For a single band pair $c, v$ the intermediate field expression for $\mathrm{d} \varepsilon$ in terms of general energy band structure defined by $E_{\mathrm{cv}}(\mathbf{k})$, the interband energy at $\mathbf{k}$, is given by

$$
\begin{aligned}
& \mathrm{d} \varepsilon^{i j}(\mathbf{F}, \Gamma, E) \cong\left(i e^{2} \hbar^{2} \mathbf{P}_{\mathrm{vc}}^{i} \mathbf{P}_{\mathrm{cv}}^{j} / \pi^{2} m^{2} E^{2}\right) \int_{\mathbf{B . Z} .} \mathrm{d}^{3} k \\
& \times \int_{0}^{\infty} \mathrm{d} s e^{-\Gamma s} \exp \left\{i\left[E_{\mathrm{cv}}-E(\mathbf{k}) s\right]\right\}\left\{\exp \left[-i(\hbar \Omega)^{3} s^{2} / 3\right]-1\right\},
\end{aligned}
$$

with $e^{-\Gamma s}$, life-time broadening term. This is an integral of an Airy convolution expression. After explicit integration [using $(\hbar \Omega)^{3} \cong F^{2}$ and $|\hbar \Omega|<\Gamma / 3$ ] the equation reduces to (Aspnes 1973)

$$
\begin{aligned}
\mathrm{d} \varepsilon^{i j}(\mathbf{F}, \Gamma, E) & \cong\left(2 e^{2} \hbar^{2} \mathbf{P}_{\mathrm{vc}}^{i} \mathbf{P}_{\mathrm{cv}}^{j} / \pi^{2} m^{2} E^{2}\right) \int_{\mathrm{B} . \mathrm{Z} .} \mathrm{d}^{3} k\left[(\hbar \Omega)^{3} /\left(E_{\mathrm{cv}}-E-i \Gamma\right)^{4}\right] \\
& \cong\left[(\hbar \Omega)^{3} / 3 E^{2}\left(\mathrm{~d}^{3} / \mathrm{d} E^{3}\right)\left[E^{2} \varepsilon^{i j}(\Gamma, E)\right]\right. \\
& \cong\left(e^{2} \hbar^{2} \mathbf{F}^{k} \mathbf{F}^{l} / 24 E^{2}\right)\left(\mu^{-1}\right)^{k l} \cdot\left(\mathrm{d}^{3} / \mathrm{d} E^{3}\right)\left[E^{2} \varepsilon^{i j}(\Gamma, E)\right]
\end{aligned}
$$


Thus the field induced change $\mathrm{d} \varepsilon$ is related to third derivative of the unperturbed $\varepsilon$ when the field is low (Aspnes 1974), i.e. $|\hbar \Omega|<\Gamma / 3$ as shown above. $\mathrm{d} \varepsilon$ and experimentally measured relative change in reflectivity $\mathrm{d} R / R$ can be related as (Aspnes 1973)

$$
\mathrm{d} R / R=\operatorname{Re}\left[C_{\mathrm{s}} C_{\mathrm{ex}} C_{\mathrm{in}} e^{i} e^{j} \mathrm{~d} \varepsilon^{i j}\right],
$$

with $C_{\mathrm{s}}=\left[2 n_{\mathrm{s}} / n\left(\varepsilon-\varepsilon_{\mathrm{a}}\right)\right]=(\alpha-\mathrm{i} \beta) ; C_{\mathrm{ex}}=\left[1-g(\varepsilon-1)^{2}\right]$, electron-hole Coulombinteraction in the contact exciton approximation and $g \leqslant 0$, strength parameter which can be assumed to be constant for a given structure

$$
C_{\text {in }}=-2 i k \int_{-\infty}^{0} \mathrm{~d} z^{\prime} e^{-2 k z^{\prime}}\left[F\left(z^{\prime}\right) / F(0)\right]^{2},
$$

a factor due to spatial dependence, or inhomogeneities, in the perturbing field; and $e^{i}$ and $e^{j}$, Cartesian components of the unit polarization vector $e$.

\subsection{Interpretation of the ER spectra}

The line shape of the low field ER is third-derivative like (Aspnes 1973) when compared to the unmodulated reflectance spectra. The presence of two well-defined extrema (one maximum and one minimum with respect to a base line, see figure 1) on a third derivative like curve is the general property which enables the determination of critical point energy to high accuracy (Aspnes 1972). Since the third derivative lineshapes are characterized by the presence of strongly enhanced critical point structure and strongly suppressed background effects, the spectral features are well localized in energy, allowing nearly degenerate critical points to be resolved.

Low-field lineshape of ER spectra in general exhibits a maxima and a minima for each critical point as shown in figure 1 (inset). The general property

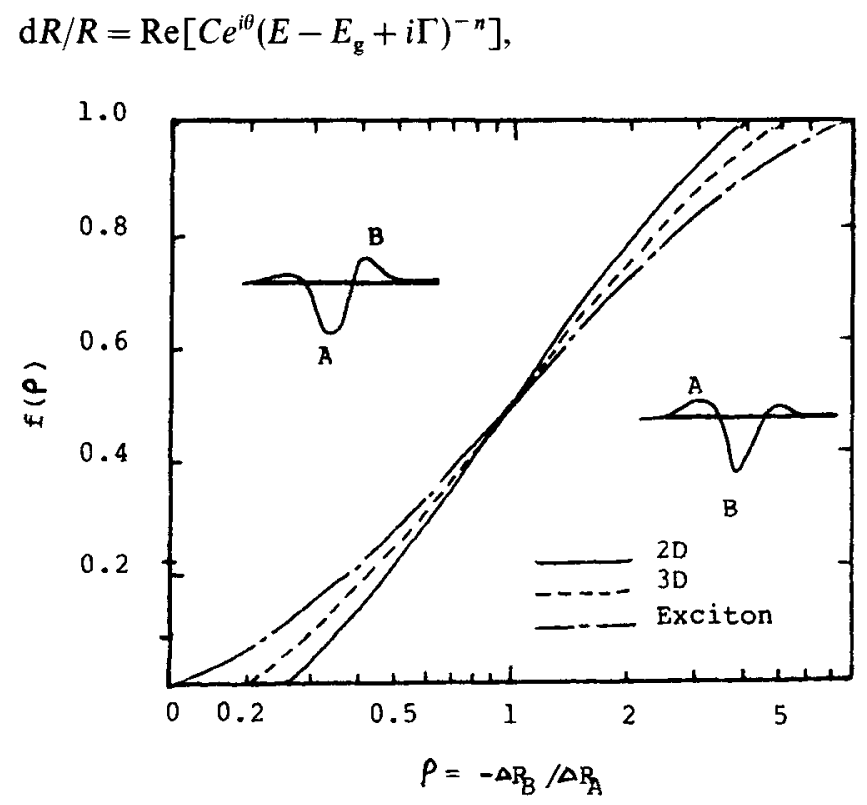

Figure 1. The function $f(\rho)$ necessary to determine the critical point energy $E_{\mathrm{g}}$ vs $\rho$ (Aspnes 1973). 
with $C$ and $\theta$, amplitude and phase-factors respectively that vary slowly with $E$ and $n \leqslant 2 ; E_{\mathrm{g}}$, energy location; and $\Gamma$, width of the structure. The three-point method, based on the lower energy extremum $\mathrm{A}$ with coordinate at $\left(\mathrm{d} R / R_{\mathrm{A}}, E_{\mathrm{A}}\right)$, and the higher energy extremum $\mathrm{B}$ with the coordinates at $\left(\mathrm{d} R / R_{\mathrm{B}}, E_{\mathrm{B}}\right)$, and the base line $(\mathrm{d} R / R=0)$, helps simplify a curve fitting process. According to this method, let

$$
\rho=\left(\mathrm{d} R / R_{\mathrm{B}}\right) /\left(\mathrm{d} R / R_{\mathrm{A}}\right)>0,
$$

be the asymmetry parameter. The curve for $f(\rho)$ vs $\rho$ is given in figure 1 . Then the energy gap $E_{\mathrm{g}}$ is given by (Aspnes 1973)

$$
E_{\mathrm{g}}=E_{\mathrm{A}}+\left(E_{\mathrm{B}}-E_{\mathrm{A}}\right) f(\rho)
$$

$E_{\mathrm{g}}$ is the most important parameter for many applications especially for materials whose $\varepsilon$ is not known. $E_{\mathrm{g}}$, according to the 'three-point-method', is located between or at one of the extrema and thus the band gap $E_{\mathrm{g}}$ can be directly calculated from the ER spectrum of the material under study. One advantage with three-point method is that it is independent of phase and amplitude (Aspnes 1980). Assuming that the 'three-pointmethod' can be applied to organic thin films the band gaps can be calculated. It has been shown that the organic materials, such as phthalocyanines, have multiband system (Xavier and Goldsmith 1995).

\section{Experimental}

Thin films may be prepared in one of the possible ways: vacuum sublimation, molecular beam epitaxy, liquid epitaxy, sputtering, Langmuir-Bridgett technique, etc. The experimental arrangements for electrolyte electroreflectance is described here.

Samples are prepared by evaporating the material in vacuum (at a temperature below the decomposing temperature) onto an (aluminum, gold, silver, etc) electrode

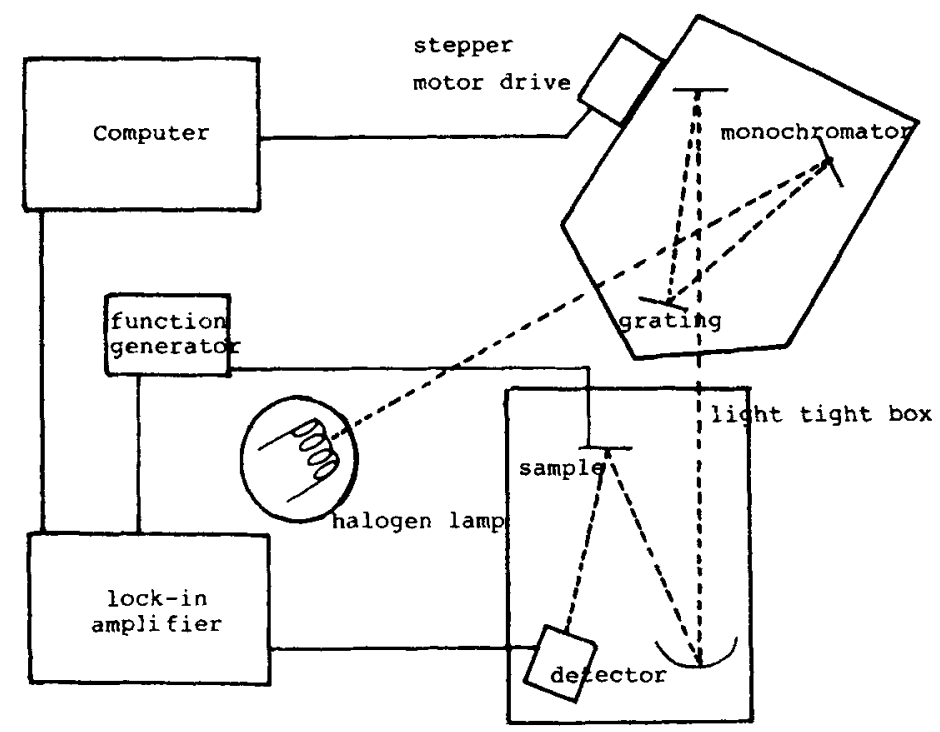

Figure 2. Experimental arrangement for the electrolyte electroreflectance measurement of organic thin films (Poras 1993). 
which has been deposited onto microscope slide (or any preferred substrate). The thickness of the film is, for better results, to be of the order of the incident wavelength of the photon (i.e. about $100 \mathrm{~nm}$ ). The electrode is isolated by an appropriately distributed layer of paraffin. The electrolyte consists of ethylene glycol containing a trace of dry $\mathrm{KOH}$. Care should be taken to avoid contamination of the electrolyte by moisture, since its presence causes the sample to be short circuited and ethylene glycol has affinity to water (Xavier 1993).

Samples for electromodulation can also be prepared by Schottky barrier method. To prepare the Schottky barrier, a thin, semi-transparent layer of gold can be evaporated onto the front surface of the sample (Poras 1993). Then the electric field is applied between the evaporated gold surface and the open surface of the thin film sample.

The experimental arrangement is as shown in figure 2. It consists of a quartz-halogen lamp focused through a pair of quartz-lenses onto the entrance slit of a monochromator. The output of the monochromator was directed by a concave mirror onto the sample which was contained in a quartz cylinder filled with the ethylene glycol--KOH solution. The container is cylindrical to assure the normal incidence of the optical excitation. A platinum electrode is inserted into the solution to complete the electrical circuit. Square wave modulation with peak-to-peak amplitude between 2 and $16 \mathrm{~V}$ at a frequency of $235 \mathrm{~Hz}$, may be applied across the sample from a function generator. (The effect of dc bias on the spectrum could also be studied by applying a dc potential between $\pm 6 \mathrm{~V}$ in series with the modulation signal in order to investigate the

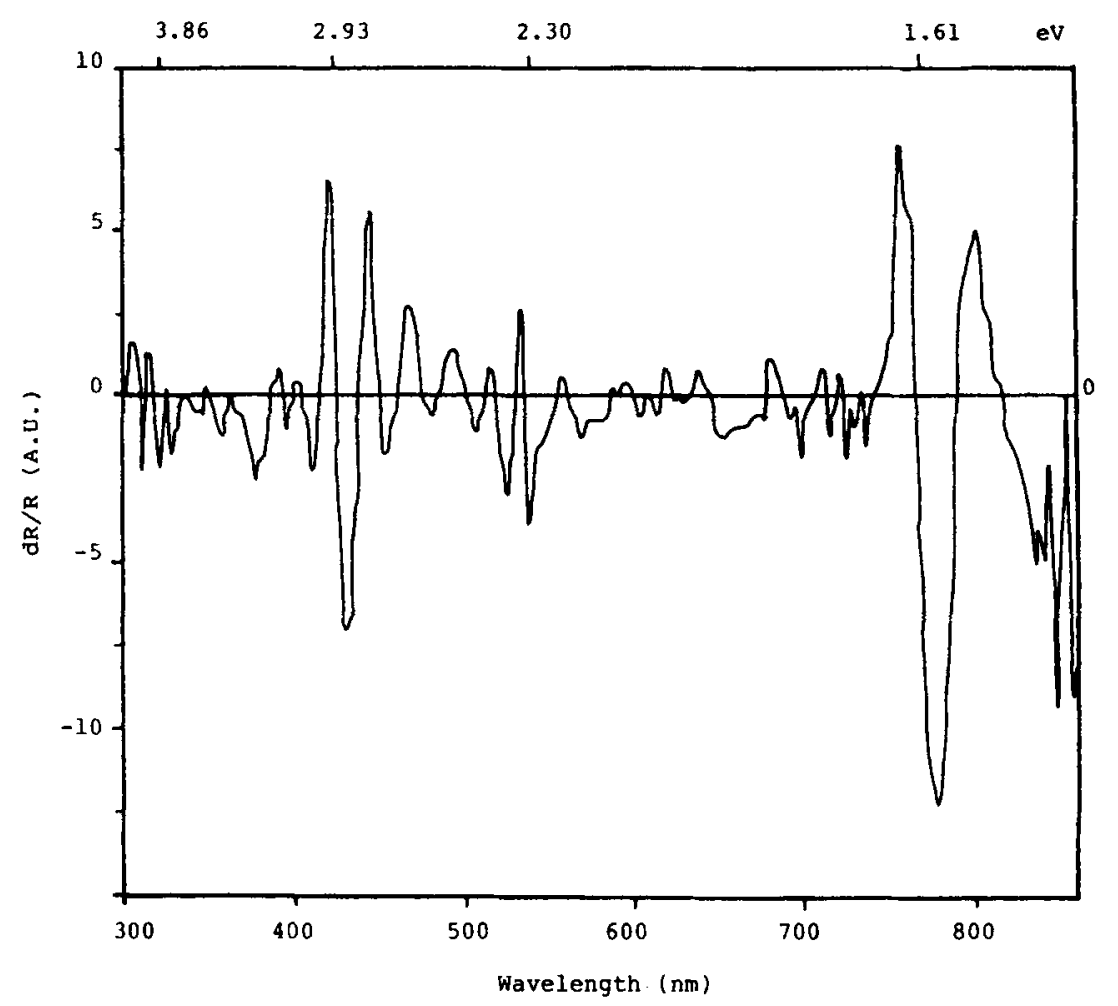

Figure 3. Electroreflectance spectrum of metal-free phthalocyanine. 


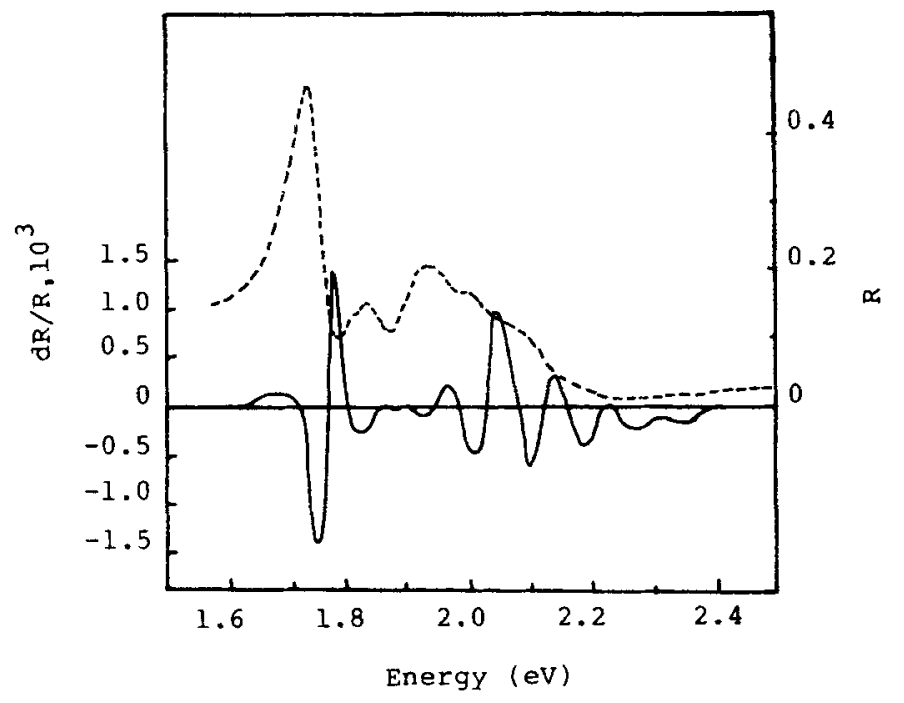

Figure 4. Ordinary reflectance $R$ (dotted curve) and electroreflectance $\mathrm{d} R / R$ (solid curve) of copper phthalocyanine single crystals (Tokura et al 1983).

Franz-Keldysh oscillations). The light reflected from the sample is focused onto a photomultiplier tube. The output of the photomultiplier tube is measured by means of a lock-in amplifier, the output of which is directed to a computer which synchronously operates with the wavelength drive of the monochromator. A servo system maintains the relative intensity of the dc component of the photomultiplier output by appropriately varying its dc potential. As a result the measured output is proportional to $\mathrm{d} R / R$. An appropriate filter could be used to eliminate the second order spectrum in measurements at the red end of the spectrum (Xavier 1993). When the film is relatively transparent, the probe beam penetrates to the interface between the film and the (glass) substrate and then is reflected outward making at least two passages through the film. The spectrum, therefore, is more probably characteristics of electroabsorption (Blinov et al 1970) rather than electroreflection.

The ER spectrum of metal free phthalocyanine film is given in figure 3. It contains prominent features between 1.5 and $1.7 \mathrm{eV}$; between 2.3 and $2.4 \mathrm{eV}$; and between 2.8 and $3 \mathrm{eV}$. These prominent features are ascribed, using Aspnes three-point method, to band-to-band transitions at critical points, viz. $1 \cdot 61,2 \cdot 30,2 \cdot 93 \mathrm{eV}$. The oscillatory features between 2.5 and $2.7 \mathrm{eV}$ are Franz-Keldysh oscillations (FKO) (Xavier and Goldsmith 1995). Figure 4 indicates the ER reflectance measured on copper phthalocyanine single crystals compared with the ordinary reflectance.

Since transitions at critical points are molecular the ER spectra for both film and single crystal are expected to be the same. This is the case when comparing the ER spectra for CuPc thin film (figure 6) with that for CuPc single crystal (figure 4). The small shifts are understood to be due to grain boundaries in the case of thin films. And the minor differences in critical points between $\mathrm{H}_{2} \mathrm{Pc}$ and $\mathrm{CuPc}$ spectra can be due to the metal ion ( $3 \mathrm{~d}$ electrons) in the case of $\mathrm{CuPc}$ in the centro symmetrical position of the Pc molecule. 


\section{Electroreflectance spectrum analysis}

Depending on the relative strength of electric field, viz. low, medium, or high fields, various information about the thin films could be understood by analyzing the ER spectrum of the material.

\subsection{Low field}

In the low field region, there is no built-in dc field and the line shape is third-derivative like and this can be used to calculate the transitions at critical points. Since the organic materials are known to have multi-band system, corresponding to each band-to-band transition there is one third-derivative line shape (Xavier and Goldsmith 1995). Using Aspnes' three-point-method $E_{\mathrm{g}}$ can be determined as mentioned above.

The results of ER spectra analysis compares well with photoconductivity analysis of Pcs spectra. In the case of $\mathrm{H}_{2} \mathrm{Pc}$ the critical points calculated by ER spectrum on thin films are $1.61,2.30$ and $2.93 \mathrm{eV}$ which correspond to the photoconductivity maxima $1 \cdot 64,2.29$ and $2.93 \mathrm{eV}$. For CuPc the ER spectra indicates the critical points to be 1.63 , 2.04 and $3.2 \mathrm{eV}$ which can be compared with the photoconductivity maxima $1.59,2.39$ and $2 \cdot 92 \mathrm{eV}$ (Xavier and Goldsmith 1995).

\subsection{Intermediate field}

When the range is intermediate, i.e. $e F a_{0} \ll E_{\mathrm{g}}$ (where $F$ is the applied electric field and $a_{0}$, the lattice constant) the dielectric function exhibits FKOs. Under the influence of the applied electric field the energy bands of a semiconductor are tilted and there is a greater probability of an electron being found inside the energy gap or the tunneling probability of an electron is increased. Thus the valence electron could tunnel through a triangular barrier in order to appear in the conduction band and the height of this tilted triangular barrier is given as

$$
d=E_{\mathbf{g}} / e F
$$

with $d$, thickness of the barrier (Pankove 1971). Further as the electron tunnels through the barrier, if it interacts with an incident photon the effective width of the barrier becomes smaller resulting in transmission becoming exponential. This is indicated as oscillatory features in reflection spectra especially when $\mathrm{d} R / R$ vs energy data are measured (Pollak and Shen 1993). These are known as FKOs (Franz 1958; Keldysh 1958). Aspnes and Studna (1973) have shown that the extrema of the modulated dielectric function resemble a sinusoidal function which could be expressed, for the $n$th extremum, as

$$
n \pi=\phi+(4 / 3)\left[\left(E_{n}-E_{\mathrm{g}}\right) / \hbar \theta\right]^{3 / 2},
$$

where $E_{n}$ is photon energy of the $n$th extremum with $n=1,2,3$, etc (Pollak and Shen 1993). And hence a plot of $\left\{4 /(3 \pi)\left[\left(E_{n}-E_{\mathrm{g}}\right)\right]^{3 / 2}\right\}$ vs the index number $n$ yields a straight line with slope $(\hbar \theta)^{3 / 2}$. And $|\hbar \theta|$ is the electro-optic energy. The ER spectrum of metal free phthalocyanine (figure 3 ) exhibit oscillatory features with extrema at $2 \cdot 44,2 \cdot 54$, $2 \cdot 69$, and $2 \cdot 81 \mathrm{eV}$ and the corresponding values of $\left\{4 /(3 \pi)\left[\left(E_{n}-E_{\mathrm{g}}\right)\right]^{3 / 2}\right\}$ with $n$ are 
Table 3. Number of extremum $(n)$ and $\left\{4 /(3 \pi)\left[\left(E_{n}-E_{\mathrm{g}}\right)\right]^{3 / 2}\right\}$.

\begin{tabular}{lc}
\hline $\begin{array}{l}\text { No. of } \\
\text { extremum }\end{array}$ & $\left\{4 /(3 \pi)\left[\left(E_{n}-E_{\mathbf{g}}\right)\right]^{3 / 2}\right\} \mathrm{eV}$ \\
\hline 1 & 0.02 \\
2 & 0.05 \\
3 & 0.10 \\
4 & 0.15 \\
\hline
\end{tabular}

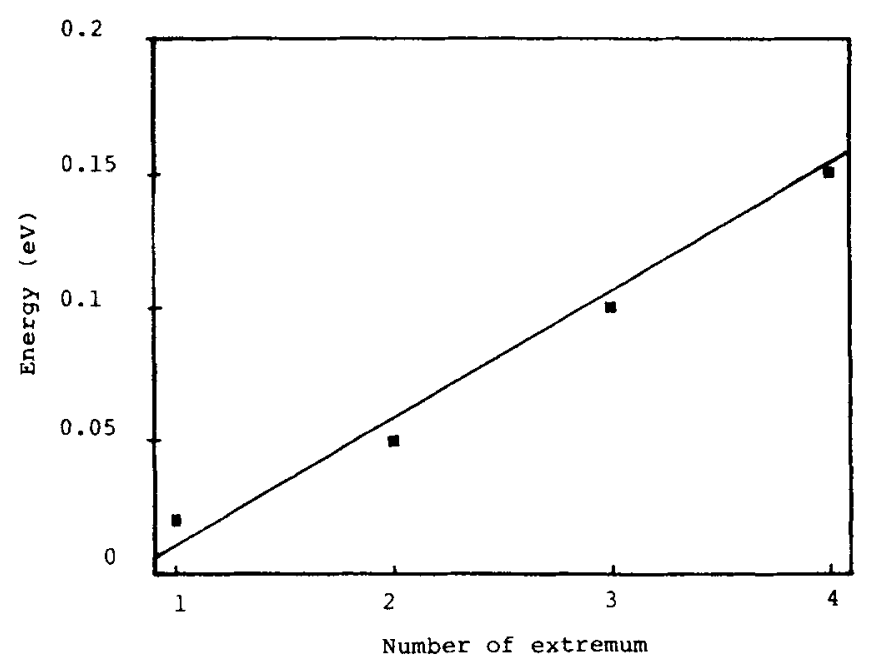

Figure 5. Number of extremum $(n)$ vs $\left\{4 /(3 \pi)\left[\left(E_{n}-E_{g}\right)\right]^{3 / 2}\right\} \mathrm{eV}$ in Franz-Keldysh oscillations of metal-free phthalocyanine thin films.

given in table 3 and the corresponding plot (figure 5) gives a straight line as discussed above.

The Franz-Keldysh oscillations are dc bias dependent. The ER spectrum of copper phthalocyanine is shown in figure 6 . In the absence of the dc bias the major features $(1.5$ to $1 \cdot 7 ; 1.9$ to $2 \cdot 1$; and 2.9 to $3.3 \mathrm{eV})$ persist while the oscillatory features on the higher energy side of $2.04 \mathrm{eV}$ flatten out (figure 7). The prominent features correspond to transition energies at the critical points, viz. $1 \cdot 63,2 \cdot 04$, and $3.20 \mathrm{eV}$ (Xavier and Goldsmith 1995). These energies are identified by Arulselvi and Xavier (1996) as the intra-molecular excitation and generation of charge carriers (Tokura et al 1983), while the FKO correspond to the inter-molecular transport of charge carriers. The FKO are inter-molecular (due to band bending) since they are dc bias dependent (figures 6 and 7) whereas the intra-molecular transition, corresponding to the critical points, are not bias-dependent. And further the critical points compare well with the photoconductivity maxima $(\$ 4.1)$ and hence the critical points are intra molecular.

\section{2a Carrier and dopant concentration: Since}

$$
(\hbar \theta)=e^{2} \hbar^{2} F^{2} / 2 \mu
$$




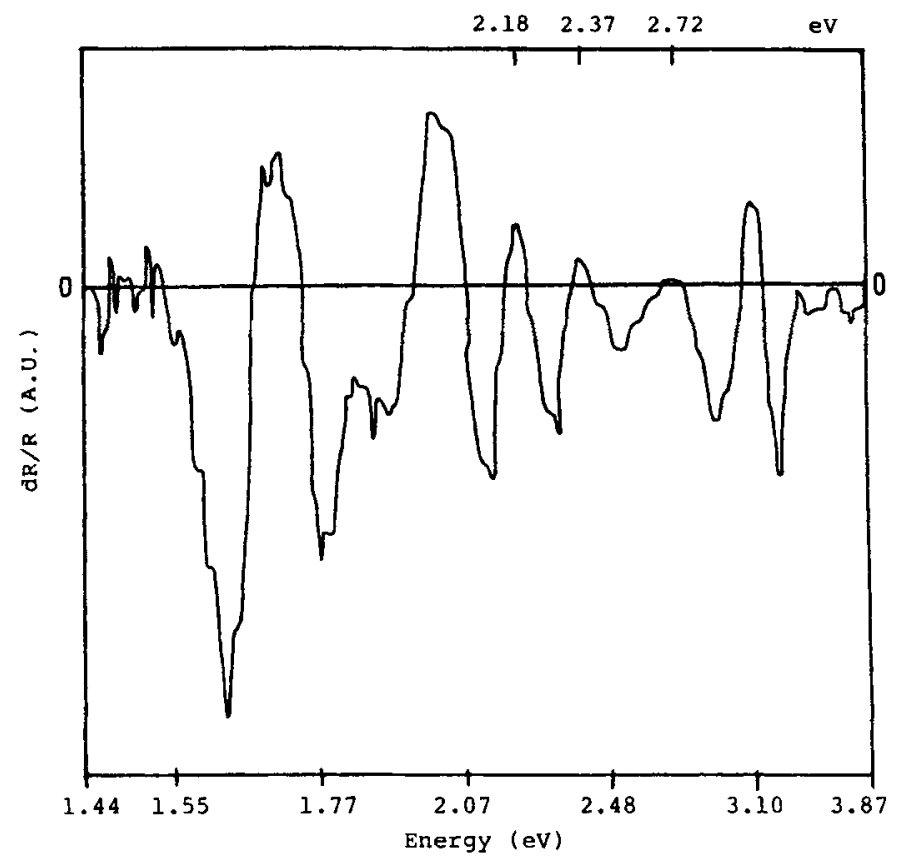

Figure 6. Electroreflectance spectrum of CuPc thin film.

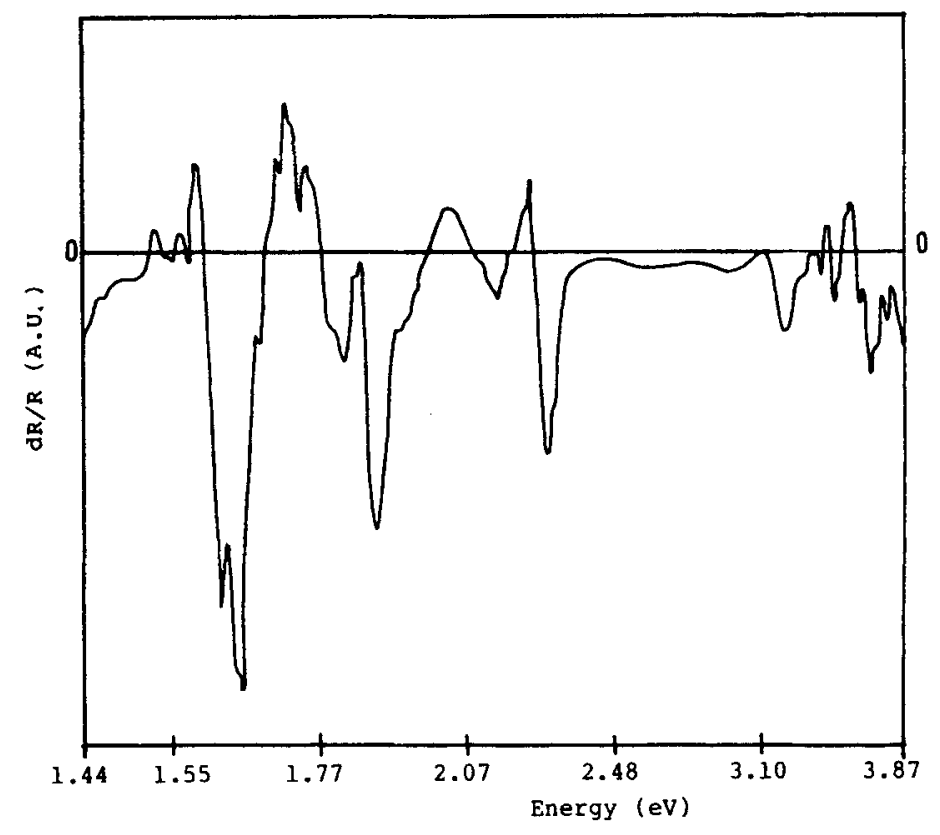

Figure 7. Bias dependence of electroreflectance of $\mathrm{CuPc}$ thin film.

(with $\mu$ as the reduced interband mass in the direction of the field), the actual electric field effective at the molecular site of the film could be determined. The plot in figure 5 between $\left\{4 /(3 \pi)\left[\left(E_{n}-E_{\mathrm{g}}\right)\right]^{3 / 2}\right\}$ vs $n$ is linear, the electric field can be accurately 
evaluated and also the built-in potential. Once the surface electric field $(F)$ is evaluated, the net carrier concentration of a semiconducting material can be estimated by plotting the dc field (from the period of FKOs) as a function of applied bias voltage (Pollak and Shen 1993). The magnitude of the electric field is related to the applied and built-in potential by

$$
F^{2}=2 e\left(N_{\mathrm{D}}+N_{\mathrm{A}}\right)\left[V_{\mathrm{B}}-V_{\mathrm{A}}-(k T / e)\right] /\left(\varepsilon \mu_{0}\right),
$$

with $N_{\mathrm{D}}$, and $N_{\mathrm{A}}$, the electron and hole carrier concentrations respectively; $V_{\mathrm{B}}$ and $V_{\mathrm{A}}$, the built-in and applied potential; $k$, Boltzman constant; $\varepsilon$, the dielectric constant and $\mu_{0}$, the permittivity of free space. A plot of the square of the field strength as measured from the FKO period from ER measurement vs the applied potential is a straight line whose slope is proportional to the dopant concentration (Poras 1993). The dopant concentration in Pcs determined by different methods are of the order of $10^{13} \mathrm{~cm}^{-3}$. According to Pollak and Shen (1993) the doping concentration has been determined to be of the order of $10^{13} \mathrm{~cm}^{-3}$ by ER method.

\subsection{High field}

In the high-field range the electro-optic energy can be given as $e F a_{0} \approx E_{\mathrm{g}}$. In this region Stark shifts are produced (Pollak and Shen 1993).

\subsection{Impurity/traps effects}

The FKO occurs on the higher energy side of the band-edge. And the structures in ER on the lower energy side of the band-edge may be attributed to impurities (Pollak and Shen 1993). This is very useful in the case of thin films of organic semiconductors since it is almost impossible to prepare very pure samples without impurities. The impurity scattering may serve to broaden the lineshape of transitions. Glembocki et al (1985) observed a correlation between the below-gap ER structure and impurity and suggested that impact ionization is the mechanism which allows impurity transition in ER.

These impurities and many defects, such as crystal imperfections, dislocations, grain boundaries, surface of the material, etc (Gutmann and Lyons 1981) act as physical or chemical traps whose density could be in the range $10^{15}-17^{15} \mathrm{~cm}^{-3}$ (Mort and Pai 1976). These impurities may be neutral molecules that cannot be separated from the host material or they could be species that resulted due to the reactivity of the material. Since these impurities are different in shape and in electronic structure they will interact with the neighbouring molecules of the host lattice in a way which is different from the interactions between a host molecule and other surrounding molecules. Thus the host molecules in the vicinity of an impurity have altered energies and positions different from those of the normal host lattice (Wright 1989). The trap density is so great that instantaneous retrapping in dark is possible. Electrons or holes may also be trapped because of the molecular vibrations within a molecule (Simon and Andre 1985). The depth of impurity and trap level could also be determined by use of photoreflectance spectrum from the frequency dependence of the photoreflectance signal (Herman 1990; Pollak and Shen 1993).

Grain boundaries, especially in thin films, lead to potential build up as barrier between the grains. And consequently photoexcitation may affect subsequent 
transport across these grain boundaries by (i) increasing the concentration of free carriers throughout the material; (ii) decreasing the interaction potential height by changing the charge density in the intergrain states; and (iii) increasing the possibility of tunneling through the intergrain built-up barriers by decreasing the depletion layer width in the adjacent grain (Bube 1992).

\section{Conclusion}

It has been shown that how electroreflectance spectrum could be applied for the organic semiconductors especially for the thin films to determine accurately the band-gaps using Aspnes three-point method; and from the analysis of FKOs, how the intra- and inter-molecular aspects of charge conduction mechanism could be explained and the carrier concentration could also be determined. Though electroreflectance method has been devised originally for inorganic semiconductors, it could be applied to the organic materials provided the following correspondence or correlation is made in the assumption, viz. atomic concept in the inorganic semiconductor is replaced by molecular aspect regarding the transport of the charge carriers. But it is to be noted that the initial excitation and absorption of charge carriers takes place within the molecule (i.e. intra-molecular). And hence the organic or molecular semiconductors possess 'dualism' regarding conduction mechanism since they exhibit both molecular as well as solid-state mechanism in their transport properties.

\section{References}

Aoyagi Y et al 1971 J. Phys. Soc. Jap. 31164

Arulselvi A and Xavier F P 1996 unpublished

Aspnes D E 1966 Phys. Rev. 147554

Aspnes D E 1972 Phys. Rev. B5 4022

Aspnes D E 1973 Surf. Sci. 37418

Aspnes D E 1974 Phys. Rev. B10 4228

Aspnes D E 1980 Modulation spectroscopy/electric field effects on the dielectric function of semiconductors, in Handbook on semiconductors (ed) M Balkanski (Amsterdam: North-Holland) Vol. 2

Aspnes D E and Studna A A 1973 Phys. Rev. B7 4605

Aspnes D E et al 1968 Phys. Rev. 166921

Aspnes D E et al 1972 Phys. Rev. $\mathbf{B 5} 4022$

Aviksoo J and Reinot T 1992 Mol. Cryst. Liq. Cryst. 217147

Baughman R H et al 1977 J. Chem. Phys. 66401

Blinov L M et al 1970 Sov. Phys. - Solid State 121246

Bordas J 1976 Some aspects of modulation spectroscopy in layer materials, in Optical and electrical properties (ed.) P A Lee (Dordrecht-Holland: Reidel)

Bube R H 1992 Photoelectronic properties of semiconductors (Cambridge: Cambridge University Press)

Callaway J 1963 Phys. Rev. 130549

Cardona M, Shaklee K L and Pollak F H 1967 Phys. Rev. 154696

Chamberlain G A 1983 Solar Cells 847

Eley D D 1989 Mol. Cryst: Liq. Cryst. 1711

Evans B L 1976 Optical properties of layer compounds, in Optical and electrical properties (ed) P A Lee, (Dordrecht-Holland: Reidel)

Franz W 1958 Z. Naturforsch. a13 484

Gamo K et al 1968 J. Phys. Soc. 25431

Glembocki O J, Bottka N and Furneaux J E 1985 J. Appl. Phys. 57432

Gutmann F and Lyons L E 1981 Organic semiconductors (Part A) (Malabar: Krieger) 
Heilmeier G H et al 1963 J. Chem. Phys. 38163

Herman M H 1990 Proc. Soc. Photo-Optical Instrum. Eng. 391286

Hsu C et al 1974 J. Chem. Phys. 614640

Inabe T, Marks T J, Burton R L, Lyding J W, McCarthy W J, Kannewurf C N, Reisner $G$ M and Herbstein F H 1985 Solid State Commun. 54501

Inokuchi H 1989 Mol. Cryst. Liq. Cryst. 17123

Kanemitsu Y et al 1991 J. Appl. Phys. 697333

Karl N 1989 Mol. Cryst. Liq. Cryst. 17131

Keldysh L V 1958 Sov. Phys. JETP 34788

Kivelson S et al 1983 Phys. Rev. B28 7236

Minami N et al 1987 Jap. J. Appl. Phys. 261754

Mort J and Pai D M 1976 Photoconductivity and related phenomena (New York: Elsevier)

Moser F H and Thomas A L 1963 Phthalocyanine compounds (New York: Reinhold)

Orti E et al 1988 J. Chem. Phys. 891009

Orti E et al 1990 J. Chem. Phys. 921228

Pankove J I 1971 Optical processes in semiconductors (Dover: New York)

Pollak F and Shen H 1993 Mater. Sci. Eng. R10 275

Poras H 1993 Optical and electro-optical properties of epitaxial GaAs deposited on a semi-insulating GaAs substrate, PhD thesis, Boston College, USA

Qian R 1989 Mol. Cryst. Liq. Cryst. 171117

Robertson M 1935 J. Chem. Soc. 615

Robertson M 1936 J. Chem. Soc. 1195

Seraphin B O et al 1966 Phys. Rev. 145628

Simon J and André J-J 1985 Molecular semiconductors (Berlin: Springer)

Schoch K F et al $1988 J$. Vac. Sci. Technol. A6 155

Streetman B G 1980 Solid state electronic devices (Englewood Cliffs: Prentice-Hall)

Tanaka T et al 1983 J. Chem. Phys. 441069

Tharmalingham K 1963 Phys. Rev. 1302204

Tokura Y, Koda T, Iyechika Y and Kuroda H 1983 Chem. Phys. Letts. 102174

Weigl J W 1977 Angew. Chemie Int. Edn. 16374

Wright J D 1989 Molecular crystals (Cambridge: Cambridge University Press)

Wrighton M S 1979 Acc. Chem. Res. 12303

Xavier F P 1993 Optical and transport properties of phthalocyanine and related compounds (Ann Arbor: UMI)

Xavier F P and Goldsmith G J 1995 Bull. Mater. Sci. 18 269, 277

Xavier F P and Pragasam J 1996 Conducting polymers (Madras: Loyola College Publications) 